\title{
Ryan : une rencontre dans l'espace figural de la souffrance
}

\author{
Audrey Lemieux \\ Université du Québec à Montréal
}

Par essence, la figure scintille. Non pas qu'elle jette de l'éclat par intervalle, comme le suggère la définition du mot " scintiller » : la figure scintille parce qu'elle ondoie et chatoie à la surface des choses, des mots, des images, avant de sombrer, pour réémerger aussitôt et replonger, et ainsi de suite, en un mouvement incessant. J'emprunte l'image du scintillement à Barthes qui, dans Le Plaisir du texte, s'en sert pour décrire la fascination qui nous prend devant la « peau qui scintille entre deux pièces (le pantalon et le tricot), entre deux bords (la chemise ouverte, le gant et la manche) ». Il ajoute que «c'est ce scintillement même qui séduit, ou encore: la mise en scène 
d'une apparition-disparition » (1973, p. 19). À la fois absence et présence, la figure semble se donner et s'esquiver dans un mouvement analogue: impossible de la fixer, de la figer, de l'empoigner; elle se dérobe sans cesse. Et pourtant, on peut l'approcher d'assez près pour la nommer, pour la dire; on pourrait même penser qu'elle nous appelle, qu'elle nous exhorte à la suivre, qu'elle nous somme de la prendre en filature. La figure fascine. Mais parfois, il arrive que le fil qui nous lie à elle ne se dévide pas et que la filature n'ait pas lieu, car, comme l'écrit François Aubral, «la figure ne se livre pas d'emblée: elle se présente, le plus souvent, sous des airs protéiformes » (1999, p. 198). Elle est donc soumise à notre attention, à notre reconnaissance surtout. La figure se donne, quoi qu'il arrive; mais pour apparaître, elle nécessite que nous suivions son mouvement, que nous la saisissions sous ses aspects les plus divers.

Au cinéma, ce processus est complexe : comment parvenir à saisir, à travers les images mouvantes, le mouvement même de la figure? Avril Dunoyer, dans une analyse consacrée à la figure de la frontière chez Jean Eustache, note que «le film n'est pas soumis à l'immobilité du tableau, et [que] les figures qui s'y dégagent [...] peuvent se constituer de plusieurs éléments dispersés aux natures différentes, qu'ils soient de l'ordre de la texture [...], de la narration, de la forme [...] » (Aubral/Chateau, 1999, p. 284). Dans Ryan, court métrage d'animation désormais célèbre de Chris Landreth, la figure de la souffrance apparaît avec une force telle qu'elle éclipse les autres figures : elle surgit, en effet, de toutes les composantes du film, c'est-à-dire tout autant des corps et des visages des protagonistes que de la composition de la bande-son, du traitement graphique des lieux, du contenu des dialogues. En fait, la figure de la souffrance est tant et si bien 
prégnante, dans Ryan, qu'elle ne fait pas seulement éclipser les autres figures, elle les engouffre. Elle serait ainsi plus que figure : elle deviendrait espace figural.

Michel Guérin, dans un texte intitulé «Idée d’une figurologie », décrit la figure comme un " complexe dynamique de métaphores » (1990, p. 23), ce qui m’amène à définir l'espace figural comme un espace où se déploierait un «complexe dynamique » de figures. On pourrait croire cet espace confiné à l'œuvre même, mais il la déborde : il s'agirait plutôt d'un espace de l'entre-deux, à mi-chemin entre un espace $\mathrm{d}^{\prime}$ « expression ${ }^{1} »-$ celui de l'artiste - et un espace de réception ${ }^{2}-$ celui que nous ménageons en nous lorsque nous entrons en contact avec une œuvre. L'espace figural serait ainsi espace de relation, espace de rencontre. Nous ne pourrions toutefois y accéder qu'à la condition de suivre le fil ténu, mais tenace, qui relie, dans une œuvre, les figures les unes aux autres. C'est d'ailleurs ce fil que j'entends suivre ici : je ferai le parcours du film Ryan en cheminant d'un motif à l'autre, d'une figure à l'autre, pour m'arrêter, enfin, sur ce que j'appelle l'espace figural de la souffrance.

\section{Motifs de la blessure et de l'agression}

Si on voulait simplifier à l'extrême le propos du film Ryan, on dirait qu'il relate la rencontre de deux cinéastes d'animation,

\footnotetext{
${ }^{1}$ Reprenant les termes de Jean-François Lyotard, Olivier Schefer définit l'espace figural comme étant l'« espace même de l'expression, [qui] exprime et désigne l'événement a-logique et a-discursif par excellence du désir » (1999, p. 919).

2 Michel Guérin souligne que la figure « appartient à la création aussi bien qu'à la réception et l'on peut dire avec autant de raison qu'on la découvre et/ou qu'on l'invente » (1986, p. 136).
} 
l'un se trouvant au début de sa carrière, l'autre ayant autrefois connu une telle déchéance qu'il en est désormais réduit à mendier dans les rues de Montréal. Mais il s'agit de bien plus que cela; il s'agit plus que d'une " histoire». Ce qui se donne d'emblée à voir, dans Ryan, n'est pas autre chose que l'« espace du dedans » des corps, espace qui a été retourné comme un gant et dont on peut apercevoir non pas les coutures et les fils mal coupés, mais diverses incarnations du motif de la blessure : déchirures, béances, autant de marques laissées par les drames intimes vécus par chacun des protagonistes. Ainsi, les lacérations colorées et lumineuses qui marquent le visage de Chris lui ont été infligées, nous explique-t-il, lorsque sa « vision romantique et débridée du monde a été définitivement démolie $^{3}$ ». Quant au trou béant et souriant qui lui déforme la tête et dans lequel la caméra nous fait chuter, il lui vient, d'une part, de ce qu'il s'est un jour trouvé dans l'incapacité de gérer ses affaires et, d'autre part, de la peur de l'échec qui s'est emparée de lui dès son plus jeune âge. Le corps et le visage de Ryan sont plus ravagés encore. Son corps est si maigre, si décharné, qu'on voit l'espace qu'il y a entre les os de ses mains, de ses bras. Sa tête est tant et si bien entamée, perforée, éclatée, qu'il ne lui reste plus qu'une portion de visage, à laquelle se ramifient des lambeaux de peau qui semblent se tenir, comme par eux-mêmes, dans le vide. Tel est le récit qu'ont tracé dans sa chair la dépendance à la cocaïne et à l'alcool, la déchéance, la dépossession, la colère.

Sur le plan pictural, le motif de la blessure altère, défait, déshumanise les corps: celui de Ryan n'a plus rien d'un

${ }^{3}$ Citation tirée des sous-titres français du film. En anglais, Chris dit : « when my unbridled romantic world view was permanently shattered ». 
organisme vivant, c'est un «corps sans organes » (Deleuze, 2002 [1981], p. 47), semblable aux anatomies distordues peintes par Francis Bacon. Assemblage de chair et de nerfs, ou plutôt de toile râpeuse et de câbles entortillés, le corps de Ryan est une machine usée, rafistolée tant bien que mal avec de la broche et des tiges de métal. De la même façon, le corps de Chris, en apparence moins brisé, n'en est pas moins une chose, au sens où l'entend Foucault :

Et puis ce corps, il est léger, il est transparent, il est impondérable; rien n'est moins chose que lui [...]. Mais jusqu'au jour où j'ai mal, où se creuse la caverne de mon ventre, où se bloquent, où s'engorgent, où se bourrent d'étoupe ma poitrine et ma gorge [...]. Alors, alors là, je cesse d'être léger, impondérable, etc.; je deviens chose, architecture fantastique et ruinée. (2009 [1966], p. 14)

Choses, machines, architectures fantastiques et ruinées: voilà qui pourrait également décrire la demi-douzaine d'éclopés qui apparaissent ponctuellement tout au long du film. Leur aspect physique n'est pas moins évocateur que ceux de Chris et de Ryan : l'un boite, l'autre a la tête difforme, un autre encore est démembré et ses jambes et ses bras ont été remplacés par des béquilles, sans compter ce personnage tout hérissé de poils, rébarbatif à tout contact avec autrui, et cet autre personnage, anéanti, dont le corps en déliquescence s'aplatit sur une table comme s'il avait été vidé de sa substance. La souffrance se livre donc sans équivoque à travers ces figures humaines certes marginales, mais surtout amoindries et meurtries. Or, l'une des singularités du film Ryan réside en ceci que la souffrance non seulement investit les corps et les visages par l'entremise du motif de la blessure, mais se matérialise, se montre en pleine action.

Le motif de l'agression fait ainsi pendant au motif de la blessure : tantôt convoquée par les souvenirs de Chris et de Ryan, tantôt suscitée par le dialogue qu'ils entretiennent, la 
souffrance assaille littéralement leurs corps. Elle apparaît sous la forme de liens colorés qui les attaquent par surprise, s'enroulent autour de leur visage et de leur corps; elle les bâillonne, les étouffe et, par le fait même, elle se donne dans son impossibilité d'être dite. La première occurrence de ce motif survient lorsque Chris nous explique, au début du film, l'origine de ses blessures. Dès l'instant où il énonce sa peur de l'échec, des bandes de couleur fondent sur son visage, dans un claquement. Le motif se répète à l'identique dans le plan suivant, qui consiste en une photo de Chris à l'âge de deux ans : de pareils liens colorés se jettent sur son visage d'enfant tandis que retentit de nouveau un bruit de claquement : l'enfant sur la photo ouvre alors la bouche et se met à pleurer.

L'une des manifestations les plus saisissantes du motif de l'agression survient sans doute au moment où Ryan se met en colère, après que Chris lui a suggéré de ne plus consommer d'alcool et de reprendre ses activités créatrices. Un long silence s'ensuit; il n'est rompu que par l'exclamation outrée de Ryan, si violente que le mur même, derrière lui, sursaute, comme s'il était fait d'une matière organique et qu'on lui avait porté un coup. La colère de Ryan s'enfle à mesure qu'il réalise qu'il lui serait impossible de se remettre à créer, ne serait-ce qu'en raison de la pauvreté matérielle dans laquelle il vit désormais. En arrièrefond, le bruit du ventilateur s'amplifie. Parvenue à son paroxysme, la colère de Ryan surgit violemment hors de son corps: lui faisant éclater la peau, de longs pics rouges lui transpercent de part en part la tête. Au même moment, tout ce qui prend place dans l'espace pictural réagit à la colère de Ryan : les éclopés se sont tus et le regardent; l'un d'entre eux se dissimule derrière une chaise, effrayé. Le mur, derrière Ryan, se distord et se brouille : une affiche qui y était épinglée se déforme, 
se liquéfie. Le micro, placé au-dessus de la tête de Ryan, perd également de sa substance, et sa couleur se mêle au magma de l'arrière-plan. En arrière-fond, le bruit du ventilateur a été remplacé par des voix déformées, torturées. Puis, tandis que Ryan s'apaise et que Chris se ressaisit, d'étranges ramures jaillissent de leurs têtes et se dressent, comme sous le coup de leurs émotions :on dirait qu'il s'agit là de leurs nerfs, non plus à fleur de peau, mais littéralement à vif. Cette séquence exemplifie la façon dont la souffrance, dans Ryan, se donne: elle y est d'autant plus prégnante qu'elle s'exprime dans le double mouvement de la blessure et de l'agression, et qu'elle déborde les corps et les visages pour affecter les objets, le lieu, la bande-son.

\section{L'entrelacs des figures : l'espace figural de la souffrance}

Si la souffrance se montre sans cesse, dans Ryan, suivant la dynamique de la blessure et de l'agression, elle n'est pourtant jamais nommée en tant que telle. Le dialogue qu'entretiennent Ryan et Chris serait ainsi semblable à la forme de leurs corps : troué, ponctionné, il est essentiellement construit autour de non-dits et de silences. Mais deux témoignages viennent combler certains des espaces vides laissés dans la trame de la vie de Ryan. Derek Lamb, ancien producteur exécutif des films de Ryan, raconte comment le cinéaste est passé d'une créativité débordante à la déchéance la plus totale, en raison de sa dépendance à la cocaïne. Le témoignage de Derek Lamb se termine tandis que l'on voit Ryan, bâillonné et ligoté par des liens de couleurs, précipité dans une chute sans fin, jusqu'à n'être plus qu'un point de fuite au centre de l'image. En guise de raccord avec le plan suivant, la main de Ryan s'abat sur la table, écrasant, du même coup, le point de fuite, son propre corps 
réduit à néant. L'air désemparé, il murmure :《ah, Jeez », puis il garde le silence jusqu'à la fin de la séquence. Le silence compense peut-être ici pour le trop-plein de ce qu'il y aurait à dire, le surcroît d'une souffrance criante. Il est peut-être aussi une façon de signifier l'impossible à dire.

L'autre témoignage nous vient de Felicity, que Ryan décrit comme l'amour de sa vie. Elle nous apprend que Ryan a toujours été instable et fragile sur le plan émotionnel. Elle raconte que le père de Ryan était un homme violent; elle raconte également que Ryan a perdu son frère dans des circonstances tragiques, quand il était jeune, et que cela l'a beaucoup affecté. Pendant que Felicity parle, le visage de Ryan est figé. Or, tout de suite après que Felicity a dit combien Ryan avait été troublé par les événements survenus dans sa jeunesse, l'image se défige, mais seul l'œil de Ryan bouge: il se ferme, accompagné d'un bruit sourd, qui nous laisse entendre combien tous ces souvenirs sont lourds de sens, lourds à porter. Puis, très rapidement, Ryan amène la conversation ailleurs : il révèle à Felicity qu'il regrette de n'avoir pas eu d'enfant avec elle. Le coq-à-l'âne semble ici jouer le même rôle que le silence dans l'exemple précédent: il masque la souffrance, l'évacue complètement.

De ces exemples se dégagent deux figures constitutives de l'espace figural de la souffrance. Dans un premier temps, la façon dont le film est construit nous incite à rapprocher les silences et les non-dits du discours non seulement des trous creusés dans les corps et les visages des personnages, mais également des manques évoqués dans l'histoire personnelle de Ryan, notamment ce vide laissé, dans son existence, par l'incapacité de créer - Ryan parle d'ailleurs de l'interruption 
de ses activités créatrices comme d'un long silence. Quand on considère, au surplus, la récurrence du motif de la chute, ce néant dans lequel sont happés tour à tour Chris et Ryan, il y a tout lieu de dire que la figure du vide est l'une des figures importantes du film. Dans un second temps, le procédé du coqà-l'âne, en tant qu'il masque autre chose, brouille la piste engagée par une idée principale, semble se donner de la même façon que ces lieux et ces corps à moitié effacés, brouillés, que présente le film à différents moments. Comme si, sous le régime de la figure de la disparition, l'effacement du discours, tout comme la quasi-dématérialisation des corps, pouvait enrayer momentanément la souffrance. Comme si, en effaçant le lieu, un ailleurs plus apaisant pouvait apparaître.

Mais parler des figures du vide et de la disparition, dans Ryan, ne peut se faire sans considérer une autre figure, celle de la mort, qui ne devient saillante que vers la fin du film et qui a elle-même pour corollaire une autre figure, celle de l'échec. Il a déjà été fait mention de cette scène où Ryan se met en colère à la suite d'une suggestion de Chris. Une fois l'explosion de colère passée, on entend la voix de Chris, en focalisation interne, qui se demande pourquoi il a fait part à Ryan de son désir de le voir renoncer à sa consommation d'alcool et recommencer à créer. On voit Chris lever les yeux vers Ryan : de son point de vue, Ryan nous apparaît sous la forme d'un squelette. La voix de Chris, encore en focalisation interne, dit : " Je te regarde et je vois plein de choses de ma mère. ${ }^{4}$ " Une série de photos de Barbara, mère de Chris, défilent alors; plusieurs voix s'entrechoquent pour dire ce qui est arrivé à Barbara: son

\footnotetext{
${ }^{4}$ Citation tirée des sous-titres français du film. En anglais, Chris dit : « I look at you and I see a lot of things about my mother. »
} 
alcoolisme, son échec personnel, ses peurs, sa colère, sa mort. Un fondu enchaîné nous fait ensuite passer de l'œil de Barbara à l'orbite du squelette de Ryan. Dans le plan suivant, Chris se trouve de nouveau devant le squelette de Ryan. Sa voix, toujours en focalisation interne, dit : « Je vois bien comme elle a baissé et je vois comment tu en es arrivé là. ${ }^{5}$ »Cette révélation ne fait que confirmer ce dont nous avons été témoins tout au long du film : Ryan prend, pour Chris, figure de l'échec, figure de la mort. Il met Chris en contact tout à la fois avec sa propre peur - celle de l'échec - et sa propre douleur, associée à l'échec personnel de sa mère qui s'est soldé, comme on l'apprend, par sa mort. Suivant cette lecture, on réalise à rebours que la mort est partout présente dans Ryan, à commencer par les blessures qui marquent les corps et qui ne sont pas autre chose que le signe d'une disparition à venir, ou plutôt d'une disparition en train d'advenir et qui creuse sa place, à même la chair, instant après instant. Quant à la figure de l'échec, intrinsèquement liée, par son caractère mortifère, à la figure de la mort, elle est aussi lisible à travers les autres figures dont il a été question : régissant les motifs de la blessure et de l'attaque, elle pousse à la chute, elle invite au vide, elle incite à la disparition. Mais on peut difficilement considérer chacune de ces figures séparément. Elles forment plutôt un entrelacs, un réseau duquel elles sont inextricables; elles ne peuvent être saisies autrement que dans leur totalité, c'est-àdire, dans ce cas-ci, l'espace figural de la souffrance.

Le figural, écrit Olivier Schefer, "fait sens sans faire histoire : quelque chose est à voir et à comprendre qui ne peut

\footnotetext{
5 Citation tirée des sous-titres français du film. En anglais, Chris dit : «I see the way that she went downhill and I see the way that you've come to this point. »
} 
se dire mais seulement se montrer » (1999, p. 916). Dans Ryan, la souffrance n'est jamais nommée en tant que telle, mais certains motifs, tels ceux de la blessure, de l'agression et de la chute, nous la font reconnaître. Cependant. les figures principales du film, c'est-à-dire celles du vide, de la disparition, de l'échec et de la mort, se livrant dans un mouvement continu, dans un entrechoquement perpétuel, ne nous permettent, à aucun moment, de dire : «voici de la souffrance». Pour reprendre les propos de François Aubral, la figure - et, par extension, l'espace figural - « ne se limite [pas] à une simple extériorité révélant et constituant la forme des choses, leur dehors, mais prend la dimension de l'intériorité, du dedans " (1999, p. 199). Qui plus est, la figure, selon Michel Guérin, est « irréductible au percept comme au concept» (1990, p. 13). Par conséquent, la souffrance, ni directement perceptible, ni même pensable, ne peut être que ressentie. Ce n'est donc pas du dehors qu'elle surgit: elle nous empoigne de l'intérieur, à la condition, toutefois, que nous acceptions d'être touchés, émus par elle.

Et comme l'espace figural est un espace de relation, son existence repose sur une autre condition: il faut, préalablement, que la démarche de l'artiste ait été traversée par le désir de toucher, d'émouvoir. On pourrait dire qu'il s'agit là d'un terrain quelque peu glissant. Or, parler du désir de toucher, d'émouvoir qui traverse une œuvre, ce n'est pas présumer de l'intention de l'artiste ou du «message» qu'il cherche à livrer, mais plutôt être attentif aux traces de son investissement affectif dans l'œuvre même. Dans Ryan, ce désir est sensible, ne serait-ce que dans cette séquence adressée aux spectateurs, au tout début du film: sous prétexte de nous fournir quelques explications, Chris Landreth nous ouvre alors, 
tout simplement, l'espace figural de son film. C'est donc à la fois une attention et une invitation; il ne nous reste plus qu'à $y$ répondre, c'est-à-dire consentir — ou non — à être fasciné par cet espace figural dont nous entrevoyons déjà les motifs et les figures. Et si jamais nous hésitions à y répondre, il se pourrait bien que ce soit par la suite l'investissement affectif du cinéaste qui nous y gagne, investissement tel qu'il transparaît dans la façon même dont Chris regarde Ryan: à travers le jeu des champs/contre-champs, sa sollicitude, son empathie, en même temps qu'elles s'adressent à Ryan, s'adressent à nous et empreignent notre propre regard.

\section{L'expérience figurale}

Trop souvent, nous avons tendance à occulter la « communauté » (Audi, 2003, p. 170) qui existe entre l'artiste et l'amateur d'art (lecteur, spectateur, visiteur) pour ne considérer que le phénomène de la création ou, à l'inverse, (le plus souvent) le phénomène de la réception. Comprendre l'expérience figurale ne peut être possible si l'on maintient ce clivage entre les pratiques; une telle perspective implique nécessairement d'imaginer, à l'instar du philosophe Michel Henry, que «celui qui regarde une forme éprouve le même pathos que celui qui l'a conçue» (Audi, 2003: 170). Devant Ryan, ce que nous voyons nous fait mal.

L'espace figural n'est pas, on l'aura compris, l'espace où se déploient les formes, l'espace où elles se détachent $\mathrm{du}$ fond pour s'offrir à notre vue. Espace de relation, espace de rencontre, l'espace figural est le lieu d'un partage (sans objet), d'un dialogue (muet), lieu où l'on répond silencieusement à une 
parole lancée sans espoir de réponse. Selon François Aubral et Dominique Chateau, il faut envisager toute figure comme un «fort activateur de pensée et de création» $(1999$, p. 3). Au contact d'une œuvre, ce n'est donc pas ce que le récit relate ou ce que la forme montre qui compte, mais bien ce que nous avons à dire à propos de ce qui émerge de ce récit ou de cette forme. La figure fait éprouver et, conséquemment, elle fait dire, elle fait écrire. L'expérience figurale, au delà de la réception d'une œuvre, est donc une expérience de création au sens où l'entend Heidegger: "être réceptif à l'art, c'est revivre l'expérience du créer » (Audi, 2003, p. 74).

Le fil que j'ai suivi jusqu'à présent, je me rends compte que je n'ai pas fait que le laisser se dévider, je l'ai tissé, je l'ai laissé prendre forme, devenir autre chose qu'un fil. Toute lecture est ainsi faite, trame singulière, à laquelle je peux seule, en tant que lectrice, donner une épaisseur, un motif. Cette trame est nécessairement celle d'un ouvrage incomplet, car chercher à comprendre les images par l'entremise d'une analyse figurale, c'est en accepter le mouvement même, accepter que subsiste toujours en chaque œuvre et en son entour une part d'absence et qu'il n'y ait pas de mots pour combler le néant qui l'environne et qui, parfois, l'envahit. La saisie des figures qui traversent une œuvre serait ainsi toujours partielle (et partiale). Barthes, dans Le Plaisir du texte, rappelle que "nous ne lisons pas tout avec la même intensité de lecture » $(1973$, p. 20). Peut-être en va-t-il de même devant un film, peut-être ne voyons-nous pas tout avec la même intensité de regard. C'est sans doute pourquoi certaines figures nous fascinent tandis que d'autres nous aveuglent, et que d'autres encore passent sous nos yeux inaperçues. Mais il importe surtout de retenir que, lorsque les figures fascinent, 
l'envoûtement qu'elles exercent ne nous pétrifie pas. $\mathrm{Au}$ contraire, il nous mobilise: les figures ne nous font pas seulement éprouver leur séduction, elles nous donnent envie, à notre tour, de la faire éprouver.

\section{Bibliographie}

Aubral, François et Dominique Chateau (dir.). (1999), Figure, Figural, Paris, L'Harmattan.

Audi, Paul. (2003), L'«Ivresse de l'art: Nietzsche et l'esthétique, Paris, Librairie générale française, coll. «Le livre de poche: biblio essais ».

BARThes, Roland. (1973), Le Plaisir du texte, Paris, Seuil, coll. « Points ».

DELEUZE, Gilles. (1981), Francis Bacon : Logique de la sensation, Paris, Seuil, coll. «L'ordre philosophique ».

FouCAUlT, Michel. (2009 [1966]), " Le Corps Utopique », dans Le Corps Utopique, suivi de Les Hétérotopies, Fécamp, Nouvelles éditions Lignes, p. 9-20.

GuÉRIN, Michel. (1986), «La figure de l'œuvre », dans Qu'est-ce qu'une œuvre?, Arles, Actes Sud, p. 112-143.

GuÉRIN, Michel. (1990), "Idée d'une figurologie», dans La Terreur et la pitié, 1- La terreur, Arles, Actes Sud, p. 11-42.

LANDRETH, Chris. (2004), Ryan, 13 minutes 57 secondes, son, couleur, v.o., s.t.f. 
SCHEFER, Olivier (1999). "Qu'est-ce que le figural? », Critique, $n^{\circ} 630$, novembre, p. 912-925.

\title{
Résumé
}

Je me propose, dans cet article, de suivre le fil ténu - mais tenace - qui relie, dans une œuvre, les figures les unes aux autres. L'œuvre que j'entends parcourir de cette façon est le court métrage Ryan, de Chris Landreth: j'y cheminerai en passant d'un motif à l'autre (la blessure et l'agression), d'une figure à l'autre (figures du vide, de la disparition, de l'échec et de la mort), pour m'arrêter, enfin, sur ce que j'appelle l'espace figural de la souffrance : espace intersubjectif où la souffrance, ni directement perceptible, ni même pensable, ne peut être qu'éprouvée.

\begin{abstract}
In this paper, my purpose is to follow the fine but strong thread that links, in an artwork, figures together. The artwork I will explore in that way is a short film from Chris Landreth, Ryan. From a pattern to another (injury and aggression), from a figure to another (figures of emptiness, disappearance, failure and death), my analysis will finally end on what I call a "figural space of suffering": intersubjective space where suffering, neither perceptible to us, nor thinkable, may only be felt.
\end{abstract}

